\title{
O.S.P.
}

L'orientation scolaire et professionnelle

$38 / 4 \mid 2009$

Sentiments d'efficacité personnelle et orientation scolaire et professionnelle - 2

\section{Hommage à Jacques Lévine}

Tribute to Jacques Lévine

Ginette Francequin

\section{CpenEdition}

Journals

Édition électronique

URL : http://journals.openedition.org/osp/2363

DOI : $10.4000 /$ osp.2363

ISSN : 2104-3795

Éditeur

Institut national d'étude du travail et d'orientation professionnelle (INETOP)

Édition imprimée

Date de publication : 15 décembre 2009

Pagination : $567-572$

ISSN : 0249-6739

Référence électronique

Ginette Francequin, « Hommage à Jacques Lévine », L'orientation scolaire et professionnelle [En ligne], 38/4 | 2009, mis en ligne le 08 décembre 2009, consulté le 23 octobre 2020. URL : http:// journals.openedition.org/osp/2363; DOI : https://doi.org/10.4000/osp.2363

Ce document a été généré automatiquement le 23 octobre 2020

(c) Tous droits réservés 


\section{Hommage à Jacques Lévine}

Tribute to Jacques Lévine

\section{Ginette Francequin}

1 Paris, le 23 mai 2009 - Salle Monnerville, Palais du Luxembourg. Jeanne Moll, Présidente de l'AGSAS ${ }^{1}$, a rassemblé autour d'Hélène Lévine des psychologues et des pédagogues pour rendre un hommage à Jacques Lévine, psychanalyste, décédé le 23 octobre 2008. Il avait inspiré des enseignants, des médecins, des rééducateurs, des psychologues scolaires et des conseillers d'orientation-psychologues pour analyser leurs pratiques professionnelles. Ginette Francequin a répondu à cette invitation pour représenter l'INETOP, où elle a pu partager durant plusieurs années des temps de travail avec Jacques Lévine et des collègues.

2 Nous retranscrivons ici le discours prononcé par Ginette Francequin en cette occasion.

3 Un jour, j'ai demandé à Lévine « Jacques, dites-moi ce que vous auriez aimé faire si vous n'étiez pas psychanalyste ? C'est la conseillère d'orientation qui vous le demande ». Il prit alors un air inspiré pour me dire :

Oh, j'ai été résistant; j'ai travaillé avec Wallon; je suis devenu docteur en psychologie ; j'ai bien connu Piaget, Chiland, mais j'aurais pu et j'aurais peut-être aimé être clochard. Oui, clochard. Tout dépend des défaites qu'un enfant vit, de ses sentiments de vide et de son désir au droit d'exister.

4 Ceci m'a suffisamment intriguée pour me donner envie de suivre les séminaires qu'il animait, ceux de «soutien au soutien » ou groupes Balint-enseignants, et ce pendant une dizaine d'années, jusqu'au moment où j'ai décidé de faire une thèse en psychologie, moi aussi. Ensuite nous nous sommes retrouvés à l'Institut national d'études du travail et d'orientation professionnelle, devant les étudiants, en jury d'examens, et dans divers colloques entre Paris et La Rochelle. 
Jacques aimait faire partager ses recherches sur les difficultés scolaires et sur la place de la psychologie à l'école, souligner les rencontres riches avec les enseignants de collège, ceux de technologie qui lui racontaient par exemple que :

On croit que l'homme qui porte un bleu de travail ne pense pas et que celui qui porte une blouse blanche réfléchit [...]. Beaucoup de jeunes découvrent l'utilité du théorème de Pythagore, à genoux, en montant brique à brique des murs à angle droit, alors que d'autres auront d'abord besoin de théoriser avant de découvrir les applications pratiques de ce théorème.

6 Je m'en souviens bien car j'ai moi-même travaillé avec plaisir avec des élèves de LEP et j'ai été choquée par les rapports et les écarts entre blouses bleues et blouses blanches.

7 Pour Lévine, ce qui donne l'envie d'apprendre, c'est la fierté d'avoir fabriqué un objet devant lequel quelqu'un du métier pourra dire: «Mais, qui a fait ça?» La reconnaissance sera aussi issue de toute l'importance de l'intelligence concrète et des réflexions menées sur les concepts d'inconscient et d'imaginaire que Jacques déclinait, en s'appuyant sur les situations exposées.

Il s'attaquait toujours au trop grand investissement sur l'abstraction à l'école, valorisant les méthodes actives. Il rappelait souvent Freinet. Il disait aussi que « apprendre avait un sens très concret qui s'est perdu : acquérir des outils pour lutter contre l'adversité et rendre la vie plus vivable ». Il valorisait une pédagogie naturelle de proximité avec les métiers, celle qui a longtemps tenu lieu d'école au sens fort du mot, " une école de métiers », comme celle que nous développons au CNAM, où il a d'ailleurs enseigné plusieurs années.

9 Jacques Lévine s'étonnait aussi d'observer que trop peu d'enseignants savent restituer les élans émotionnels, les étonnements, les démarches conquérantes ou dépressives qui ont fait vibrer les découvreurs et les savants. Il disait que l'« on ne tient pas assez les enfants au courant des toutes dernières découvertes qui se font dans le monde en matière de médecine, d'aviation, d'architecture ».

10 Ses auditeurs à l'INETOP aimaient ses enthousiasmes et ses révoltes. Les auditeurs de ses séminaires ont apprécié son opposition récente à l'étiquetage des enfants de moins de trois ans, à la suppression des RASED, et son rappel : «C'est manquer d'agressivité qui serait grave! Tous les enfants au Moi solide en ont, pour se faire respecter ».

1124 ou 25 Octobre 2008. Un message sur ordinateur m'annonce la mort de Jacques. Machinalement, j'ouvre mon courrier qui est sur le bureau. La grosse enveloppe contient le dernier ouvrage de Jacques Lévine, le titre est à son image: L'enfant philosophe, avenir de l'humanité?, édité aux ESF dans la collection dirigée par Philippe Meirieu. Il l'a fait, ce livre auquel il tenait tant! Jacques a toujours eu le goût de transmettre et, ici, ce sont dix ans de réflexion sur les ateliers de philosophie. Je me souviens alors qu'en 2004, il m'avait dit avec contentement devoir intervenir au Parlement de la Communauté française à Bruxelles sur le monde philosophique de l'enfant. Il était heureux aussi car un instituteur avait apprécié ses ateliers de philosophie pour la classe : ils étaient innovants.

12 Enfin, on sort des " $\mathrm{Y}$ a qu'à les faire taire, $\mathrm{Y}$ a qu'à revenir aux bonnes vieilles méthodes, et $Y$ a qu'à les exclure » écrit Philippe Meirieu, en préface du livre, associant le travail de Lévine à celui des pédagogues tels Pestalozzi, Korczak, Montessori pour développer l'idée que l'enfant philosophe « accède à l'humaine condition, capable de se penser lui même et de se penser dans le monde ». 
13 L'expérience des Ateliers de Philosophie a commencé en 1996, « dans le respect de la singularité de chacun et l'aspiration à l'universalité: l'intime et l'universel se répondent, s'appellent réciproquement", puis elle s'est poursuivie par celle des Ateliers de Psychologie et d'Interrogation Collective qui font une part plus importante au débat. Les jeunes du cycle 3 de l'école primaire et les collégiens sont invités à examiner les points d'accord et de désaccord à propos de deux sortes de questions, «Pensez-vous que...?» et «Comment expliquer que...?», sur des sujets aussi importants que « Le bonheur », « L'amour », « La haine ».

14 Lévine pensait (et nous étions d'accord avec lui pour le penser) que tous les enfants rencontrent très tôt les questions complexes et les vivent pleinement : la solitude, le bonheur, la justice, le pouvoir, la mort, la peur d'être aimé et d'être dévoré par les autres. Ils se posent la question du sens de leur existence et celui de la présence des hommes dans le monde. Leur vie psychique est intense et il est essentiel qu'ils puissent en débattre pour s'ouvrir au monde. Pour cela, Les ateliers de philosophie se sont inspirés de recherches internationales et des travaux de Matthew Lipman et de Tozzi, mais ici il s'est agi de monter les ateliers selon trois objectifs :

- Faire participer les enfants aux débats sur les questions essentielles à la vie et à la civilisation.

- Faire l'expérience de sa propre capacité à produire de la pensée sur des questions importantes pour l'humanité (la question fondamentale des origines que pose tout enfant dès trois ans).

- Apprendre à intérioriser la pensée : ce qui se passe dans le silence est aussi important que ce qui se dit.

"C'est quoi un adulte?", «Pourquoi on meurt? " Les réponses à ces questions vont montrer deux choses: d'abord qu'une relation au savoir comporte du cognitif et des découvertes vraies et, ensuite, qu'un autre statut du Moi identitaire est possible, car l'enfant a besoin d'une relation sur un mode "équivalent » à celui dont l'adulte lui donne l'exemple.

Avant de s'engager dans les ateliers, Jacques Lévine avait posé trois préambules :

- L'enfant est un être génétiquement philosophe (il est producteur, porte-parole et bénéficiaire de la pensée, comme le disent Deleuze, Eiguer ou Pichon-Rivière).

- L'enfant est un être génétiquement anthropologue (le regard originaire est d'abord une confrontation au réel, à l'étant là ; ensuite le Moi-Monde est désir d'accès au savoir, interrogation, expérience, pour retrouver la base du vivre ensemble). Dickens, Malot, Pinocchio, Peter Pan sont aussi essentiels, rappelle Lévine, que Les origines de la pensée de Wallon, La représentation du monde des enfants de Piaget, Le petit Hans de Freud ou la Petite Piggle de Winnicott.

- L'enfant est un être génétiquement métaphysicien : il a l'intelligibilité de ce qui se joue ; chaque voyage au pays des idées est traversé par le «pareil que prévu » ou l'« autrement que prévu », selon qu'il mène ses voyages comme Phileas Fogg, Robinson Crusoé ou Rembrandt.

Comme en psychanalyse, pour cette pédagogie, Jacques Lévine pose un cadre, un temps, une circulation de paroles, une place pour l'enseignant, parle du sens du silence, il propose un thème (par exemple: «La beauté ? ", "La peur ? ", « Le courage ? ", " La honte ? ", « La joie ? ", « Être intelligent », "L'amour ? », « La famille », « Le corps ? »). C'est cohérent, cela ressemble à Jacques, au cadre analytique et c'est aussi la trame de la condition humaine avec la vie et ses structures (la famille, l'école, le travail, la vieillesse, la mort) ; la vie et ses obstacles, ses valeurs (l'envie d'apprendre, la justice, 
l'égalité) ; et aussi le racisme, la violence, la maladie ; la vie et les sentiments éprouvés : le chagrin, le droit d'exister, l'espoir, la colère, la nostalgie.

Cette rencontre pour les élèves et les collégiens qu'il a créée et mis au point avec des professeurs des écoles relève d'une rencontre naturelle avec les concepts. Mais elle est souvent peu considérée en classe, par rapport au "ça parle scolaire », considéré comme la seule voie royale d'accès à la culture. Or, expliquait Jacques Lévine, il y a une pulsion (un désir et un plaisir) d'équivalence, comme une motivation à philosopher.

19 Son concept de "pulsion d'équivalence » m'intéresse beaucoup car je le trouve évident en psychologie. En effet, on parle souvent de «lutte contre les inégalités sociales » - ce qui est juste, et Lévine ne négligeait pas la dimension sociale de la vie--, mais il est nécessaire d'insister sur ce désir de reconnaissance de parole équivalente qui existe chez tout enfant. C'est le désir d'équivalence du droit à exister, d'être un interlocuteur valable, qui incite à se dépasser, à devenir responsable comme habitant de cette terre, comme citoyen.

Là était pour Lévine, l'avenir de la pédagogie. Il affirmait aussi : «L'intérêt des Ateliers que je viens d'évoquer est de faire découvrir à l'élève, quel que soit son âge, qu'il peut avoir un autre rapport au savoir que celui que l'école traditionnelle lui impose ».

21 La question est alors de comprendre pourquoi des enfants qui n'investissent pas les savoirs traditionnels éprouvent une intense satisfaction de participer à ces Ateliers. Pour répondre, on trouve le plaisir du statut d'équivalence qui est celui de découvrir «la pensée dont on est soi-même la source ». C'est de pouvoir, comme les adultes, se confronter aux problèmes qui préoccupent les hommes; c'est de pratiquer le débat dans un cadre collectif; c'est de vivre l'expérience du groupe qui «cogite ». Tout se passe comme si l'image de soi et l'expérience de la pensée devenaient plus riches, plus complexes, donc plus intéressantes.

Cette question de comprendre le manque d'investissement lié à la solitude vaut pour les enseignants qui s'étonnent de l'intérêt des réponses et de l'implication des enfants. Voilà ce que Jacques Lévine nous a appris, à nous, ses collaborateurs en éducation, en orientation, c'est l'idée urgente à faire passer que «tant qu'on n'introduira pas dans les classes une diversification capable de répondre aux différentes façons d'apprendre, de s'intéresser au monde et de grandir, on continuera de s'installer dans un procès stérile et injuste aux enfants, aux parents et aux enseignants ».

Fort de l'appui collectif, chacun en groupe du type «Balint-enseignants » recherche ce qui peut être changé, le « modifiable ». J'entends encore Jacques Lévine dire :

Le problème se pose bien dans les termes suivants : il s'agit d'inventer les stratégies et les détours qui permettront de développer des enfants, qui ne sont pas pareils au départ, qui ne seront pas nécessairement pareils ou égaux au terme du parcours, mais à qui auront été données des chances pareilles en matière de compétences diversifiées.

24 Il s'agit bien sûr aussi que l'institution scolaire reconnaisse ses limites, donne les moyens de travailler de manière nouvelle, et que nous nous autorisions à tout faire pour que chaque enfant se sente reconnu, dans la famille et à l'école, comme un être potentiellement « apportant».

Alors, pour nous aider à faire ce travail sur nous, pour nous former à la relation, il avait mis au point le «soutien au soutien", un lieu pour penser les ébranlements corporels et psychiques, un lieu et une méthode pour re-dynamiser la croissance. C'est ce que 
relate le livre de 2009, sorti après ses obsèques, Prévenir les souffrances d'école, cosigné Jacques Lévine et Jeanne Moll. Je voudrais finir en insistant sur deux bases fondamentales que me laisse le travail réalisé avec Jacques Lévine et les groupes Balint entre 1982 et 1992 puis en 2004-2006:

- Une conviction, celle de saisir que chez tout enseignant, de la maternelle à l'université, trois capacités sont essentielles: la capacité d'alliance, la capacité de réalisme, la capacité de confiance dans l'aventure humaine. Elles font partie de tout accompagnement sécurisant de l'intérieur, qui par une présence qui pose «je fais face à la vie et j'entends vos craintes » peut rassurer l'autre avec réalisme. Le réalisme, c'est amener la personne enfant, adolescent ou jeune adulte à distinguer ce qui se fait de ce qui ne se fait pas, à distinguer l'essentiel de ce qui ne l'est pas.

- Une autre manière de regarder : il semblait la préciser avec son idée de passage du « regard photo» au "regard cinéma». Le "regard photo» serait celui qui fige, qui referme la temporalité sur les difficultés. Le "regard cinéma », inscrirait l'autre dans une temporalité ré-ouverte, il donne à intérioriser que, malgré toutes les difficultés, il est possible de se vivre comme porteur du passé, porteur de son présent, mais aussi porteur d'une trajectoire qui situe et relativise passé et présent, pour que la vie puisse continuer. C'est ce qui donne, comme le disait aussi Winnicott « le sentiment que la vie vaut la peine d'être vécue ».

\section{NOTES}

1. Association des groupes de soutien au soutien.

\section{AUTEUR}

\section{GINETTE FRANCEQUIN}

est conseillère d'orientation psychologue et HDR maître de conférences en psychologie clinique et sociale, membre du Laboratoire LISE au CNAM. Courriel : ginette.francequin@wanadoo.fr 